\title{
Furstenberg and Margulis Awarded 2020 Abel Prize
}

The Norwegian Academy of Science and Letters has awarded the Abel Prize for 2020 to Hillel Furstenberg of the Hebrew University of Jerusalem and Gregory Margulis of Yale University "for pioneering the use of methods from probability and dynamics in group theory, number theory, and combinatorics."

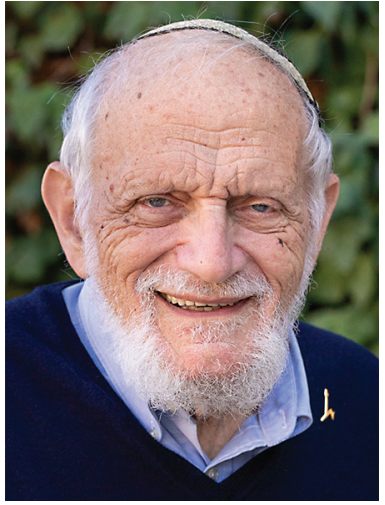

Hillel Furstenberg

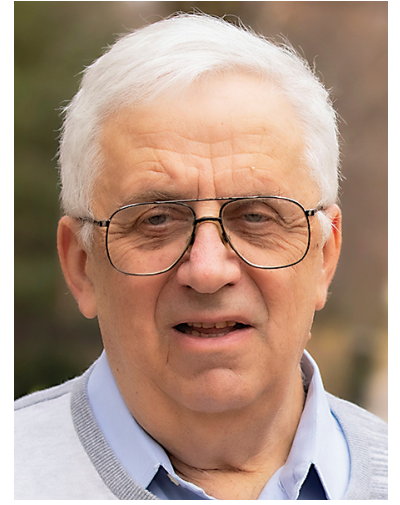

Gregory Margulis

\section{Citation}

A central branch of probability theory is the study of random walks, such as the route taken by a tourist exploring an unknown city by flipping a coin to decide between turning left or right at every cross. Hillel Furstenberg and Gregory Margulis invented similar random walk techniques to investigate the structure of linear groups, which are, for instance, sets of matrices closed under inverse and product. By taking products of randomly chosen matrices, one seeks to describe how the result grows and what this growth says about the structure of the group.

Furstenberg and Margulis introduced visionary and powerful concepts, solved formidable problems, and discovered surprising and fruitful connections between group theory, probability theory, number theory, combinatorics, and graph theory. Their work created a school of thought which has had a deep impact on many areas of mathematics and applications.

For permission to reprint this article, please contact: reprint-permission aams.org.

DOI: https://dx.doi.org/10.1090/noti 2110

Starting from the study of random products of matrices, in 1963, Hillel Furstenberg introduced and classified a notion of fundamental importance, now called "Furstenberg boundary." Using this, he gave a Poisson-type formula expressing harmonic functions on a general group in terms of their boundary values. In his works on random walks at the beginning of the 1960s, some in collaboration with Harry Kesten, he also obtained an important criterion for the positivity of the largest Lyapunov exponent.

Motivated by Diophantine approximation, in 1967, Furstenberg introduced the notion of disjointness of ergodic systems, a notion akin to that of being coprime for integers. This natural notion turned out to be extremely deep and to have applications to a wide range of areas, including signal processing and filtering questions in electrical engineering, the geometry of fractal sets, homogeneous flows, and number theory. His " $\times 2 \times 3$ conjecture" is a beautifully simple example which has led to many further developments. He considered the two maps taking squares and cubes on the complex unit circle and proved that the only closed sets invariant under both these maps are either finite or the whole circle. His conjecture states that the only invariant measures are either finite or rotationally invariant. In spite of efforts by many mathematicians, this measure classification question remains open. Classification of measures invariant by groups has blossomed into a vast field of research influencing quantum arithmetic ergodicity, translation surfaces, Margulis's version of Littlewood's conjecture, and the spectacular works of Marina Ratner. Considering invariant measures in a geometric setting, Furstenberg proved, in 1972, the unique ergodicity of the horocycle flow for hyperbolic surfaces, a result with many descendants.

Using ergodic theory and his multiple recurrence theorem, in 1977, Furstenberg gave a stunning new proof of Szemerédi's theorem about the existence of large arithmetic 
progressions in subsets of integers with positive density. In subsequent works with Yitzhak Katznelson, Benjamin Weiss, and others, he found higher dimensional and far-reaching generalizations of Szemerédi's theorem and other applications of topological dynamics and ergodic theory to Ramsey theory and additive combinatorics. This work has influenced many later developments, including the works of Ben Green, Terence Tao, and Tamar Ziegler on the Hardy-Littlewood conjecture and arithmetic progressions of prime numbers.

Gregory Margulis revolutionized the study of lattices of semisimple groups. A lattice in a group is a discrete subgroup such that the quotient has a finite volume. For semisimple groups, Margulis classified these lattices in his "superrigidity" and "arithmeticity" theorems in the mid1970s. Armand Borel and Harish-Chandra constructed lattices in semisimple groups using arithmetic constructions, essentially as the group of integer-valued matrices in a large matrix group. Margulis proved that all lattices in rank 2 or higher arise from this arithmetic construction, as conjectured by Atle Selberg. In 1978, Margulis unveiled the structure of these lattices in his "normal subgroup theorem." Central to his techniques is the amazing and surprising use of probabilistic methods (random walks, Oseledets theorem, amenability, Furstenberg boundary), as well as Kazhdan property $(\mathrm{T})$.

In his 1970 dissertation, Margulis constructed the socalled "Bowen-Margulis measure" of a compact Riemannian manifold of strictly negative variable curvature. Using the mixing property of geodesic flows with respect to this measure, he proved an analogue of the prime number theorem, an asymptotic formula for the number of closed geodesics shorter than a given length. Before this, the only such counting result was via the Selberg trace formula, which works only for locally symmetric spaces. Since then, numerous counting and equidistribution problems have been studied using Margulis's mixing approach.

Another spectacular application of his methods is the proof in 1984 of the decades-old Oppenheim conjecture in number theory: a nondegenerate quadratic form with three or more variables either takes a dense set of values on the integers or is a multiple of a form with rational coefficients.

In graph theory, Margulis's creativity resulted in his construction in 1973 of the first known explicit family of expanders, using Kazhdan property (T). An expander is a graph with high connectivity. This notion, introduced by Mark Pinsker, comes from the study of networks in communications systems. Expander graphs are now a fundamental tool in computer science and error-correcting codes. In 1988 Margulis constructed optimal expanders, now known as Ramanujan graphs, which were discovered independently by Alex Lubotzky, Peter Sarnak, and Ralph Phillips.
The influence of Furstenberg and Margulis extends way beyond their results and original fields. They are recognized as pioneers by a wide community of mathematicians, from Lie theory, discrete groups, and random matrices to computer science and graph theory. They have demonstrated the ubiquity of probabilistic methods and the effectiveness of crossing boundaries between separate mathematical disciplines, such as the traditional dichotomy between pure and applied mathematics.

\section{Biographical Sketch: Hillel Furstenberg}

Note: This biographical information is taken from the Abel Prize website: https://www.abelprize.no/c76018/binfi 1 /down 1oad.php?tid=76027

When Hillel (Harry) Furstenberg published one of his early papers, a rumor circulated that he was not an individual but instead a pseudonym for a group of mathematicians. The paper contained ideas from so many different areas, surely it could not possibly be the work of one man?

The anecdote may be apocryphal, but it illustrates a truth that has been consolidated throughout his academic life: Furstenberg has a deep technical knowledge of diverse areas and has made insightful and surprising connections between them. In particular, he has made fundamental contributions to the field of ergodic theory, which have had far-reaching applications in number theory, geometry, combinatorics, group theory, and probability.

Furstenberg was born in Berlin in 1935. His family was Jewish. A few months before the outbreak of the Second World War, they managed to get out of Germany and fled to the United States. Furstenberg's father died on the journey, and Hillel was brought up by his mother and elder sister in an orthodox community in New York.

Furstenberg became interested in mathematics when he saw his teacher getting into a muddle when explaining well-known theorems. The student enjoyed finding his own proofs. "Sometimes bad teachers do a good job!" he says. He attended the high school and college of Yeshiva University, graduating in 1955 with a BA and an MS. As an undergraduate, he was already publishing papers. "Note on One Type of Indeterminate Form" (1953) and "On the Infinitude of Primes" (1955) both appeared in the American Mathematical Monthly, the latter giving a topological proof of Euclid's famous theorem that there are infinitely many primes.

Furstenberg went to Princeton University to study for his doctorate under the supervision of Salomon Bochner. He received his $\mathrm{PhD}$ in 1958 for his thesis, "Prediction Theory." When it was published in 1960, one reviewer said: "The work stands as a first-rate and highly original dissertation on a very difficult subject."

After spending a year as an instructor at Princeton and one at MIT, he got his first job as an assistant professor in 1961 at the University of Minnesota. In a series of articles, 
beginning in 1963 with "A Poisson Formula for SemiSimple Lie Groups," he continued to establish himself as a groundbreaking thinker. His work showing that the behavior of random walks on a group is intricately related to the structure of the group-which led to what is now called the "Furstenberg boundary" - has been hugely influential in the study of lattices and Lie groups.

He was promoted to full professor at Minnesota, but in 1965 he left the United States for the Hebrew University of Jerusalem, where he stayed until his retirement in 2003.

In his 1967 paper, "Disjointness in Ergodic Theory, Minimal Sets, and a Problem in Diophantine Approximation," Furstenberg introduced the notion of "disjointness," a notion in ergodic systems that is analogous to coprimality for integers. The notion turned out to have applications in areas such as number theory, fractals, signal processing, and electrical engineering.

In his 1977 paper, "Ergodic Behavior of Diagonal Measures and a Theorem of Szemerédi on Arithmetic Progressions," Furstenberg used methods from ergodic theory to prove a celebrated result by Endre Szemerédi (Abel Prize laureate, 2012), which states that any subset of the integers with positive upper density contains arbitrarily large arithmetic progressions. Furstenberg's proof was more conceptual than Szemerédi's, and it completely changed the area. Its insights also became very fruitful, leading to important results such as the proof by Ben Green and Terence Tao that the sequence of prime numbers includes arbitrarily large arithmetic progressions.

Furstenberg's decision to spend almost all of his career in Israel helped establish the country as a world center for mathematics, in particular for ergodic theory. In the academic year 1975-1976, he ran a year-long program on ergodic theory at the Israeli Institute of Advanced Study, together with Benjamin Weiss, which is considered to have transformed the field.

Of his many accolades, Furstenberg has won the Israel Prize, regarded as the top honor in Israel, and the Wolf Prize in mathematics. He is a member of the Israel Academy and the American Academy of Arts and Sciences.

Furstenberg married Rochelle, a magazine writer specializing in arts and culture, in 1958. They have five children, sixteen grandchildren, and a growing number of great-grandchildren.

\section{Biographical Sketch: Gregory Margulis}

Note: This biographical information is taken from the Abel Prize website: https://www. abelprize.no/c76018/binfil /down1oad.php?tid=76026

In a glittering mathematical career, Gregory (Grisha) Margulis has introduced many influential ideas, solved long-standing open questions, and discovered deep connections between different mathematical fields. His signature approach has been to use ergodic theory in unexpected and ingenious ways, which has led to the creation of entirely new areas of study.

Born in Moscow in 1946, he gained international recognition at age sixteen with a silver medal at the International Mathematical Olympiad. He attended Moscow State University, receiving his $\mathrm{PhD}$ in 1970 under the supervision of Yakov Sinai (Abel Prize laureate, 2014). His dissertation revealed a particularly original mind: he constructed a measure-now called the Bowen-Margulis measure-that enabled him to discover new properties about the geometry of hyperbolic space. His methods subsequently inspired many new questions and areas of active research.

Margulis won the 1978 Fields Medal, at age only thirtytwo, for his work on lattices in Lie groups, notably his arithmeticity and superrigidity theorems. The arithmeticity theorem states that all irreducible lattices in semisimple Lie groups of rank greater than 2 are arithmetic, and the superrigidity theorem that a representation of such a lattice extends to a representation of the ambient Lie group. The superrigidity proof demonstrated novel applications of ergodic theory, establishing powerful new methods that became very influential in many fields.

Jacques Tits (Abel Prize laureate, 2008) said of Margulis in 1978: "It is not exaggerated to say that, on several occasions, he has bewildered the experts by solving questions which appeared to be completely out of reach at the time."

Margulis, however, was unable to receive the Fields Medal, since the Soviet authorities refused him a visa to attend the awards ceremony in Helsinki, Finland. He was only allowed to travel abroad in 1979 when Soviet academics were given more personal freedoms. During the 1980s, he visited academic institutions in Switzerland, France, and the United States, before settling at Yale in 1991, where he has been ever since.

Earlier in his career, Margulis faced discrimination for being of Jewish origin. Even though he was one of the top young mathematicians in the country, he was unable to find a job at Moscow University. Instead, he worked at the much less prestigious Institute for Problems in Information Transmission. His contact with colleagues at the institute, however, led to a remarkable discovery. They told him about a type of connected network called an "expander graph." Within days Margulis had constructed the first known example of an expander graph using ideas from representation theory, an abstract and seemingly unrelated field. His discovery was groundbreaking and has had many applications in computer science.

Margulis again demonstrated his knack for proving theorems in stunning and surprising ways when in 1978 he revealed what is now called his normal subgroup theorem, about lattices in Lie groups. His proof was a very original combination of the theory of amenable groups on the one hand and the Kazhdan property $(\mathrm{T})$ from representation theory on the other. 


\section{COMMUNICATION}

In 1984 he proved the Oppenheim conjecture, an idea from number theory first stated in 1929, using methods from ergodic theory. More important than the result was the whole idea of using ergodic theory in this way, and this created a new area, now called homogenous dynamics. The work of three recent Fields medalists-Elon Lindenstrauss, Maryam Mirzakhani, and Akshay Venkatesh-all builds on Margulis's earlier ideas.

Margulis has been as prolific as he has been diverse. When in 2008 the Pure and Applied Mathematics Quarterly ran an article listing Margulis's major results, it ran to more than fifty pages.

Margulis's honors include the Fields Medal (1978), the Lobachevsky Prize (1996), and the Wolf Prize (2005). He is a member of the US National Academy of Sciences and a Fellow of the AMS.

Margulis and his wife Raisa (Raya) have a son and a granddaughter.

\section{About the Prize}

The Niels Henrik Abel Memorial Fund was established in 2002 to award the Abel Prize for outstanding scientific work in the field of mathematics. It carries a cash award of 7.5 million Norwegian krone (approximately US $\$ 720,000$ ). The prize is awarded by the Norwegian Academy of Science and Letters, and the choice of Abel Laureate is based on the recommendation of the Abel Committee, which consists of five internationally recognized research scientists in the field of mathematics. The Committee is appointed for a period of two years.

Read more about the recipients' life and work, as well as a list of previous recipients of the Abel Prize, at https://www.abe1prize.no/c76018/seksjon/vis .htm1?tid=76019\&strukt_tid=76018.

-From announcements of the Norwegian Academy of Science and Letters

\section{Credits}

Photo of Hillel Furstenberg is by Yosef Adest, Hebrew University of Jerusalem.

Photo of Gregory Margulis is courtesy of Dan Rezetti.

\section{JOIN THE AMS... \\ or renew your membership today}

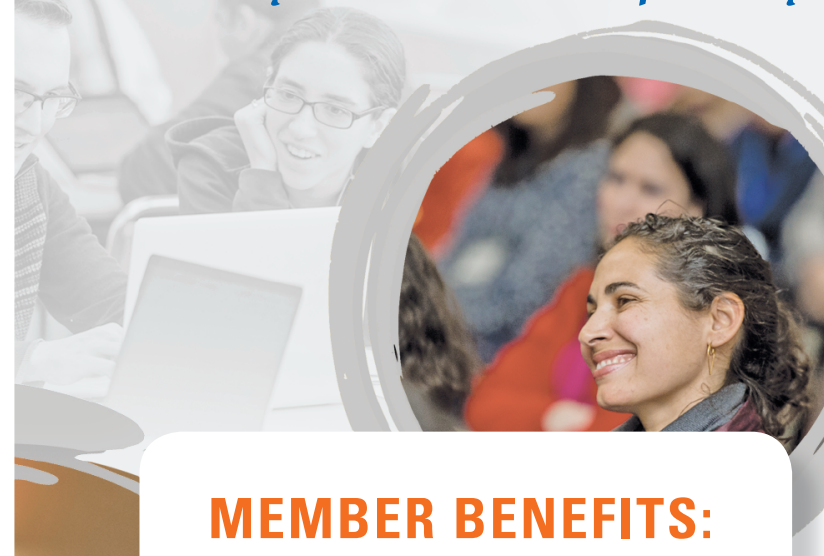

- Individual AMS members receive free standard shipping on orders delivered to addresses in the United States (including Puerto Rico) and Canada

- Discounts on AMS publications including MAA Press books

- Subscriptions to Notices and Bulletin

- Discounted registration for world-class meetings and conferences

- Access to online AMS Member Directory

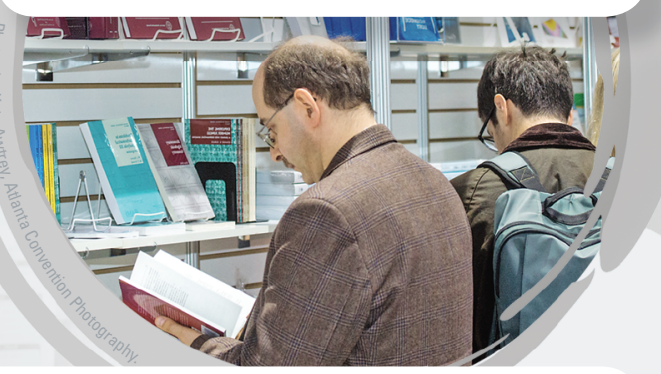

Learn more and join online at www.ams.org/membership.

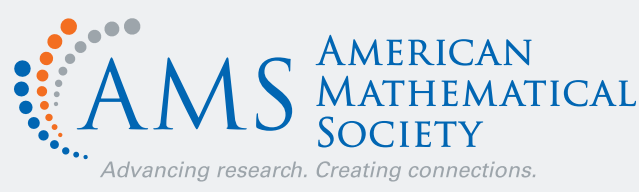

